
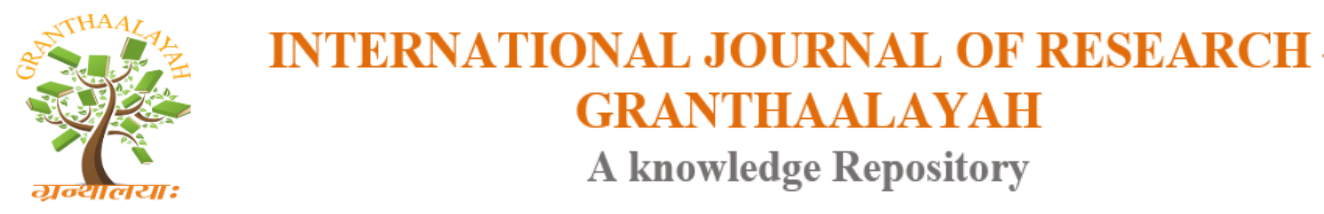

Science

\title{
A REDUCTION OF REAL POWER LOSS BY ENRICHED GENETIC ALGORITHM
}

\author{
Dr.K.Lenin *1 \\ ${ }^{* 1}$ Professor, Department of EEE, Prasad V.Potluri Siddhartha Institute of Technology, Kanuru, \\ Vijayawada, Andhra Pradesh -520007, India
}

\begin{abstract}
In this paper Enriched Genetic Algorithm (EGA) is proposed to solve the optimal reactive power problem. In order to overcome the drawbacks of standard genetic algorithm (GA) and particle swarm optimization (PSO) algorithm, some improved mechanisms based on non-linear ranking selection, competition and selection among several crossover offspring and adaptive change of mutation scaling are adopted in the genetic algorithm, and dynamical parameters are adopted in PSO. The new population is produced through three approaches to improve the global optimization performance. Proposed algorithm has been tested in standard IEEE 57 bus test system and simulation results reveal the better performance of the proposed algorithm in reducing the real power loss.
\end{abstract}

Keywords: Genetic Algorithm; Particle Swarm; Optimal Reactive Power; Transmission Loss.

Cite This Article: Dr.K.Lenin. (2018). "A REDUCTION OF REAL POWER LOSS BY ENRICHED GENETIC ALGORITHM.” International Journal of Research - Granthaalayah, 6(5), 167-176. https://doi.org/10.29121/granthaalayah.v6.i5.2018.1438.

\section{Introduction}

Reactive power optimization places an important role in optimal operation of power systems. Various numerical methods like the gradient method [1,2], Newton method [3] and linear programming [4-7] have been implemented to solve the optimal reactive power dispatch problem. Both the gradient and Newton methods have the intricacy in managing inequality constraints. The problem of voltage stability and collapse play a key role in power system planning and operation [8] Evolutionary algorithms such as genetic algorithm have been already projected to solve the reactive power flow problem [9-11]. Evolutionary algorithm is a heuristic methodology used for minimization problems by utilizing nonlinear and non-differentiable continuous space functions. In [12], Hybrid differential evolution algorithm is projected to increase the voltage stability index. In [13] Biogeography Based algorithm is projected to solve the reactive power dispatch problem. In [14], a fuzzy based method is used to solve the optimal reactive power scheduling method. In [15], an improved evolutionary programming is used to elucidate the optimal reactive power dispatch problem. In [16], the optimal reactive power flow problem is 
solved by integrating a genetic algorithm with a nonlinear interior point method. In [17], a pattern algorithm is used to solve ac-dc optimal reactive power flow model with the generator capability limits. In [18-20] proposes a two-step approach to calculate Reactive power reserves with respect to operating constraints and voltage stability. In this paper Enriched Genetic Algorithm is proposed to solve the optimal reactive power problem. Genetic algorithm (GA) is very efficient at exploring the entire search space, but it is relatively poor in finding the precise local optimal solution in the region where the algorithm converges. A new method of optimization, Particle Swarm optimization (PSO), is able to accomplish the same goal as GA optimization in a new and faster way [21-25]. Since PSO and GA both work with a population of solutions, combining the searching abilities of both methods seems to be a good approach. Some attempts have been made in this direction, but with a weak integration of the two strategies. In order to improve the speed of convergence of evolutionary algorithms, in this paper, GA and PSO are strong combined for solving optimal reactive power problem. Firstly, some improved mechanisms such as non-linear ranking selection, competition and selection among several crossover offspring and adaptive change of mutation scaling are adopted in the genetic algorithm. Then, the genetic algorithm is combined with PSO that is improved by dynamical parameters. During each iteration, the population is divided into three parts, which are evolved with the elitist strategy, PSO strategy and genetic algorithm strategy respectively. Therefore, this kind of technique can make balance between acceleration convergence and averting precocity as well as stagnation. Proposed algorithm has been tested in standard IEEE 57 bus test system and simulation results reveal the better performance of the proposed algorithm in reducing the real power loss.

\section{Problem Formulation}

Main objective of the reactive power problem is to minimize the real power loss.

\subsection{Active Power Loss}

The objective of the reactive power dispatch problem is to minimize the active power loss and can be written in equations as follows:

$\mathrm{F}=P_{L}=\sum_{\mathrm{k} \in \mathrm{Nbr}} \mathrm{g}_{\mathrm{k}}\left(\mathrm{V}_{\mathrm{i}}^{2}+\mathrm{V}_{\mathrm{j}}^{2}-2 \mathrm{~V}_{\mathrm{i}} \mathrm{V}_{\mathrm{j}} \cos \theta_{\mathrm{ij}}\right)$

Where F- objective function, $\mathrm{P}_{\mathrm{L}}$ - power loss, $\mathrm{g}_{\mathrm{k}}$ - conductance of branch, $\mathrm{V}_{\mathrm{i}}$ and $\mathrm{V}_{\mathrm{j}}$ are voltages at buses $\mathrm{i}, \mathrm{j}$, Nbr- total number of transmission lines in power systems.

\subsection{Voltage Profile Improvement}

To minimize the voltage deviation in PQ buses, the objective function (F) can be written as:

$\mathrm{F}=P_{L}+\omega_{\mathrm{v}} \times \mathrm{VD}$

Where VD - voltage deviation, $\omega_{\mathrm{v}}$ - is a weighting factor of voltage deviation.

And the Voltage deviation given by:

$$
\mathrm{VD}=\sum_{\mathrm{i}=1}^{\mathrm{Npq}}\left|\mathrm{V}_{\mathrm{i}}-1\right|
$$


Where Npq- number of load buses

\subsection{Equality Constraint}

The equality constraint of the problem is indicated by the power balance equation as follows:

$$
P_{G}=P_{D}+P_{L}
$$

Where $\mathrm{P}_{\mathrm{G}^{-}}$total power generation, $\mathrm{P}_{\mathrm{D}}$ - total power demand.

\subsection{Inequality Constraints}

The inequality constraint implies the limits on components in the power system in addition to the limits created to make sure system security. Upper and lower bounds on the active power of slack bus $\left(\mathrm{P}_{\mathrm{g}}\right)$, and reactive power of generators $\left(\mathrm{Q}_{\mathrm{g}}\right)$ are written as follows:

$$
\begin{gathered}
\mathrm{P}_{\text {gslack }}^{\min } \leq \mathrm{P}_{\text {gslack }} \leq \mathrm{P}_{\text {gslack }}^{\max } \\
\mathrm{Q}_{\mathrm{gi}}^{\min } \leq \mathrm{Q}_{\mathrm{gi}} \leq \mathrm{Q}_{\mathrm{gi}}^{\max }, \mathrm{i} \in \mathrm{N}_{\mathrm{g}}
\end{gathered}
$$

Upper and lower bounds on the bus voltage magnitudes $\left(\mathrm{V}_{\mathrm{i}}\right)$ is given by:

$$
\mathrm{V}_{\mathrm{i}}^{\min } \leq \mathrm{V}_{\mathrm{i}} \leq \mathrm{V}_{\mathrm{i}}^{\max }, \mathrm{i} \in \mathrm{N}
$$

Upper and lower bounds on the transformers tap ratios $\left(\mathrm{T}_{\mathrm{i}}\right)$ is given by:

$$
\mathrm{T}_{\mathrm{i}}^{\min } \leq \mathrm{T}_{\mathrm{i}} \leq \mathrm{T}_{\mathrm{i}}^{\max }, \mathrm{i} \in \mathrm{N}_{\mathrm{T}}
$$

Upper and lower bounds on the compensators $\left(Q_{c}\right)$ is given by:

$$
\mathrm{Q}_{\mathrm{c}}^{\min } \leq \mathrm{Q}_{\mathrm{c}} \leq \mathrm{Q}_{\mathrm{C}}^{\max }, \mathrm{i} \in \mathrm{N}_{\mathrm{C}}
$$

Where $\mathrm{N}$ is the total number of buses, $\mathrm{N}_{\mathrm{g}}$ is the total number of generators, $\mathrm{N}_{\mathrm{T}}$ is the total number of Transformers, $\mathrm{N}_{\mathrm{c}}$ is the total number of shunt reactive compensators.

\section{Hybridization of Standard Genetic Algorithm (GA) and Particle Swarm Optimization (PSO) Algorithm}

The proposed Enriched Genetic Algorithm (EGA) combines standard genetic algorithm (GA) and particle swarm optimization (PSO) algorithm to form a hybrid algorithm. Due to combination of different optimization mechanisms, not only the offspring can keep diversity, but also PSO can keep the balance of global search and local search, so the entire search ability of the algorithm can be improved.

\section{Genetic Algorithm (GA)}


Floating-point GA uses floating-point number representation for the real variables and thus is free from binary encoding and decoding. It takes less memory space and works faster than binary GA. Some practical schemes to improve GA performance are introduced in this paper. According to the optimal results, we can conclude that these measures are effective and helpful in improving convergence property and accuracy.

\section{Nonlinear Ranking Selection}

Ranking methods only require the evaluation function to map the solutions to a partially ordered set. All individuals in a population are ranked from best to worst based on their fitness values. It assigns the probability of an individual based on its rank $(r)$ and it is expressed as follows:

$\left\{\begin{array}{c}p(r)=q^{\prime}(1-q)^{r-1} \\ q^{\prime}=\frac{q}{1-(1-q)^{p}}\end{array}\right.$

Such that

$\sum_{r=1}^{P} p(r)=1$

Where

$q=$ the probability of selecting the best individual $=[0,1]$,

$r=$ the rank of the individual $=$

$\{1$, for the best individual

$\{P$, for the worst individual

$P=$ the population size

It can be seen that this selection probability doesn't use the absolute value information of fitness value so that it avoid the fitness value scale transformation and control the prematurity to some extent.

\section{Competition and Selection}

In natural biological evolution, two parents after crossover can produce several offspring, and the competition also exists among the offspring which are produced by the same parents. Motivate by this phenomenon, we adopt competition and selection among several crossover offspring. Different from the conventional algorithm in which two parents only produce two offspring, the two parents, chromosomes as $a_{s}=\left[x_{1}^{s}, x_{2}^{s}, \ldots, x_{n}^{s}\right]$ and $a_{t}=\left[x_{1}^{t}, x_{2}^{t}, \ldots, x_{n}^{t}\right]$ in this algorithm will produce four chromosomes according to the following mechanisms :

$$
\begin{aligned}
& b_{1}=\left[b_{1}^{1}, b_{2}^{1}, \ldots, b_{n}^{1}\right]=\frac{a_{s}+a_{t}}{2} \\
& b_{2}=\left[b_{1}^{2}, b_{2}^{2}, \ldots, b_{n}^{2}\right]=a_{\max }(1-\omega)+\max \left(a_{s}, a_{t}\right) \omega \\
& b_{3}=\left[b_{1}^{3}, b_{2}^{3}, \ldots, b_{n}^{3}\right]=a_{\min }(1-\omega)+\min \left(a_{s}, a_{t}\right) \omega \\
& b_{4}=\left[b_{1}^{4}, b_{2}^{4}, \ldots, b_{n}^{4}\right]=\frac{\left(a_{\max }+a_{\min }\right)(1-\omega)+\left(a_{1}+a_{2}\right) \omega}{2} \\
& a_{\max }=\left[x_{1}^{\max }, x_{2}^{\max }, \ldots, x_{n}^{\max }\right]
\end{aligned}
$$


$a_{\min }=\left[x_{1}^{\min }, x_{2}^{\min }, \ldots, x_{n}^{\min }\right]$

Where $\omega \epsilon[0,1]$ denotes the weight to be determined by users, $\max \left(a_{s}, a_{t}\right)$ denotes the vector with each element obtained by taking the maximum among the corresponding element of $a_{s}$ and $a_{t}$.Among $b_{1}$ to $b_{4}$, the two with the largest fitness value are used as the offspring of the crossover operation. As seen from Eqs. (12) to (16), the potential offspring spreads over the domain. At the same time, (12) and (16) results in searching around the centre region of the domain, (13) and (14) can move $b_{2}$ and $b_{3}$ to be near $a_{\max }$ and $a_{\min }$ respectively. Thus, the offspring generated by this operator, is better than that obtained by arithmetic crossover or heuristic crossover.

\section{Mutation}

This is the unary operator responsible for the fine tuning capabilities of the system, so that it can escape from the trap of local optimum. It is defined as follows: For a parent $p$, if variable $\mathrm{p}_{\mathrm{k}}$ was selected at random for this mutation, the result is:

$\bar{P}=\left(P_{1}, \ldots, \overline{P_{k}}, \ldots, P_{n}\right)$

$\overline{P_{k}}=\epsilon\left\{\max \left(P_{k}-\mu \frac{P_{k}^{\max }-P_{k}^{\min }}{2}, P_{k}^{\min }\right), \min \left(P_{k}+\mu \frac{P_{k}^{\max }-P_{k}^{\min }}{2}, P_{k}^{\max }\right)\right\}$

And $P_{k}^{\max }, P_{k}^{\min }$ are upper and lower bounds of $\mathrm{P}_{\mathrm{k}}$ respectively, $\mu$ decreased with the increase of iterations.

$\mu(\tau)=1-r^{[1-(\tau / T)]^{b}}$

Where $r$ is uniform random number in [0,1], T is the maximum number of iterations, $\boldsymbol{\tau}$ is the current iteration number, and $b$ is the shape parameter. From (20), at the initial stage of evolution, for small value of $r, \mu(\boldsymbol{\tau}) \approx 1$, the mutation domain is large in this case. However, in the later evolution, when $\boldsymbol{\tau}$ approaches $T, \mu(\boldsymbol{\tau}) \approx 0$, the mutation domain become small and search in the local domain.

\section{Particle Swarm Algorithm (PSO)}

The PSO conducts searches using a population of particles which correspond to individuals in GAs. The population of particles is randomly generated initially. Each particle represents a potential solution and has a position represented by a position vector $\overrightarrow{x_{l}}$. A swarm of particles moves through the problem space, with the moving velocity of each particle represented by a position vector $\overrightarrow{v_{l}}$ At each time step, a function $f_{i}$ representing a quality measure is calculated by using $\overrightarrow{x_{l}}$ as input. Each particle keeps track of its own best position, which is associated with the best fitness it has achieved so far in a vector $\overrightarrow{p_{l}}$. Furthermore, the best position among all the particles obtained so far in the population is kept track of as $\overrightarrow{p_{g}}$. At each time step $\boldsymbol{\tau}$, by using the individual best position, $\overrightarrow{p_{l}}(\boldsymbol{\tau})$ and global best position, $\overrightarrow{p_{g}}(\boldsymbol{\tau})$ a new velocity for particle $\mathrm{i}$ is updated by

$\overrightarrow{v_{l}}(\tau+1)=\omega \overrightarrow{v_{l}}(\tau)+c_{1} \emptyset_{1}\left(\overrightarrow{p_{l}}(\tau)-\overrightarrow{x_{\imath}}(\tau)\right)+c_{2} \emptyset_{2}\left(\overrightarrow{p_{g}}(\tau)-\overrightarrow{x_{\imath}}(\tau)\right)$ 
Where $\boldsymbol{c}_{1}$ and $\boldsymbol{c}_{2}$ are acceleration constants and $\emptyset_{1} \& \emptyset_{2}$ are uniformly distributed random numbers in $[0,1]$. The term $\overrightarrow{v_{l}}$ is limited to its bounds. If the velocity violates this limit, it is set to its proper limit.

$\omega$ is the inertia weight factor and in general, it is set according to the following equation:

$\omega=\omega_{\max }-\frac{\omega_{\max }-\omega_{\min }}{T} . \tau$

Where $\omega_{\max }$ and $\omega_{\min }$ is maximum and minimum value of the weighting factor respectively. $T$ is the maximum number of iterations and $\tau$ is the current iteration number. Based on the updated velocities, each particle changes its position according to the following:

$\overrightarrow{x_{l}}(\tau+1)=\overrightarrow{x_{l}}(\tau)+h(\tau) \overrightarrow{v_{l}}(\tau+1)$

Where

$h(\tau)=h_{\max }-\frac{\left(h_{\max }-h_{0}\right) \cdot \tau}{T}$

Where $h_{\max }$ and $h_{0}$ are positive constants.

According to (21) and (23), the populations of particles tend to cluster together with each particle moving in a random direction. The computation of PSO is easy and adds only a slight computation load when it is incorporated into GA. Furthermore, the flexibility of PSO to control the balance between local and global exploration of the problem space helps to overcome premature convergence of elite strategy in GAs, and also enhances searching ability. The global best individual is shared by the two algorithms, \& also it can avoid the premature convergence in PSO.

Integration of GA \& PSO for the entire run, which consists chiefly of genetic algorithm, combined with PSO, the sequential steps of the algorithm are given below;

Step 1: Randomly initialize the population of $P$ individuals within the variable constraint range. Step 2: Calculate the fitness of the population from the fitness function, and order ascendingly.

Step 3: The top $N$ individuals are selected as the elites and reproduce them directly to the next generation.

Step 4: The S individuals followed are evolved with PSO and their best positions are updated.

Step 5: The bottom individuals are evolved with GA and produce P-S-N offspring.

Step 6: Combine the three parts as the new generation and calculate the fitness of the population. Choose the best position among all the individuals obtained so far kept as the global best.

Step 7: Repeat steps 3-6 until a stopping criterion, such as a sufficiently good solution being discovered or a maximum number of generations being completed, is satisfied. The best scoring individual in the population is taken as the final answer.

\section{Simulation Results}


Proposed Enriched Genetic Algorithm (EGA) has been tested in standard IEEE-57 bus power system. The reactive power compensation buses are 18, 25 and 53. Bus 2, 3, 6, 8, 9 and 12 are PV buses and bus 1 is selected as slack-bus. The system variable limits are given in Table 1.

The preliminary conditions for the IEEE-57 bus power system are given as follows:

$\mathrm{P}_{\text {load }}=12.010$ p.u. Q $_{\text {load }}=3.011$ p.u.

The total initial generations and power losses are obtained as follows:

$\sum P_{G}=12.5501$ p.u. $\sum Q_{G}=3.3228$ p.u.

$\mathrm{P}_{\text {loss }}=0.25758$ p.u. $\mathrm{Q}_{\text {loss }}=-1.2047$ p.u.

Table 2 shows the various system control variables i.e. generator bus voltages, shunt capacitances and transformer tap settings obtained after EGA based optimization which are within the acceptable limits. In Table 3, shows the comparison of optimum results obtained from proposed EGA with other optimization techniques. These results indicate the robustness of proposed EGA approach for providing better optimal solution in case of IEEE-57 bus system.

Table 1: Variable limits

\begin{tabular}{|c|c|c|c|c|c|c|c|c|}
\hline \multicolumn{9}{|c|}{ Reactive Power Generation Limits } \\
\hline Bus no & 1 & \multicolumn{2}{|c|}{2} & 3 & 6 & 8 & 9 & 12 \\
\hline Qgmin & -1.4 & \multicolumn{2}{|c|}{-.015} & -.02 & -0.04 & -1 & -0.03 & -0.4 \\
\hline Qgmax & 1 & \multicolumn{2}{|c|}{0.3} & 0.4 & 0.21 & 1 & 0.04 & 1.50 \\
\hline \multicolumn{9}{|c|}{ Voltage And Tap Setting Limits } \\
\hline vgmin & \multicolumn{2}{|c|}{ Vgmax } & \multicolumn{2}{|c|}{ vpqmin } & \multicolumn{2}{|c|}{ Vpqmax } & tkmin & tkmax \\
\hline 0.9 & \multicolumn{2}{|c|}{1.0} & \multicolumn{2}{|c|}{0.91} & \multicolumn{2}{|c|}{1.05} & 0.9 & 1.0 \\
\hline \multicolumn{7}{|c|}{ Shunt Capacitor Limits } & & \\
\hline Bus no & \multicolumn{2}{|c|}{18} & \multicolumn{2}{|c|}{25} & \multicolumn{2}{|l|}{53} & & \\
\hline Qcmin & \multicolumn{2}{|l|}{0} & \multicolumn{2}{|c|}{0} & \multicolumn{2}{|l|}{0} & & \\
\hline Qcmax & \multicolumn{2}{|l|}{10} & \multicolumn{2}{|c|}{5.2} & \multicolumn{2}{|l|}{6.1} & & \\
\hline
\end{tabular}

Table 2: Control variables obtained after optimization

\begin{tabular}{|l|l|}
\hline $\begin{array}{l}\text { Control } \\
\text { Variables }\end{array}$ & EGA \\
\hline V1 & 1.10 \\
\hline V2 & 1.039 \\
\hline V3 & 1.028 \\
\hline V6 & 1.029 \\
\hline V8 & 1.027 \\
\hline V9 & 1.024 \\
\hline V12 & 1.019 \\
\hline Qc18 & 0.0656 \\
\hline Qc25 & 0.200 \\
\hline Qc53 & 0.0472 \\
\hline T4-18 & 1.011 \\
\hline T21-20 & 1.054 \\
\hline T24-25 & 0.879 \\
\hline T24-26 & 0.864 \\
\hline T7-29 & 1.060 \\
\hline T34-32 & 0.872 \\
\hline
\end{tabular}




\begin{tabular}{|l|l|}
\hline T11-41 & 1.021 \\
\hline T15-45 & 1.036 \\
\hline T14-46 & 0.914 \\
\hline T10-51 & 1.028 \\
\hline T13-49 & 1.062 \\
\hline T11-43 & 0.912 \\
\hline T40-56 & 0.901 \\
\hline T39-57 & 0.951 \\
\hline T9-55 & 0.951 \\
\hline
\end{tabular}

Table 3: Comparison results

\begin{tabular}{|l|l|l|l|l|}
\hline S.No. & $\begin{array}{l}\text { Optimization } \\
\text { Algorithm }\end{array}$ & Finest Solution & Poorest Solution & $\begin{array}{l}\text { Normal } \\
\text { Solution }\end{array}$ \\
\hline 1 & NLP [26] & 0.25902 & 0.30854 & 0.27858 \\
\hline 2 & CGA [26] & 0.25244 & 0.27507 & 0.26293 \\
\hline 3 & AGA [26] & 0.24564 & 0.26671 & 0.25127 \\
\hline 4 & PSO-w [26] & 0.24270 & 0.26152 & 0.24725 \\
\hline 5 & PSO-cf [26] & 0.24280 & 0.26032 & 0.24698 \\
\hline 6 & CLPSO [26] & 0.24515 & 0.24780 & 0.24673 \\
\hline 7 & SPSO-07 [26] & 0.24430 & 0.25457 & 0.24752 \\
\hline 8 & L-DE [26] & 0.27812 & 0.41909 & 0.33177 \\
\hline 9 & L-SACP-DE [26] & 0.27915 & 0.36978 & 0.31032 \\
\hline 10 & L-SaDE [26] & 0.24267 & 0.24391 & 0.24311 \\
\hline 11 & SOA [26] & 0.24265 & 0.24280 & 0.24270 \\
\hline 12 & LM [27] & 0.2484 & 0.2922 & 0.2641 \\
\hline 13 & MBEP1 [27] & 0.2474 & 0.2848 & 0.2643 \\
\hline 14 & MBEP2 [27] & 0.2482 & 0.283 & 0.2592 \\
\hline 15 & BES100 [27] & 0.2438 & 0.263 & 0.2541 \\
\hline 16 & BES200 [27] & 0.3417 & 0.2486 & 0.2443 \\
\hline 17 & Proposed EGA & 0.22106 & 0.23178 & 0.22142 \\
\hline
\end{tabular}

\section{Conclusion}

In this paper, Enriched Genetic Algorithm (EGA) successfully solved optimal reactive power problem. In order to improve the speed of convergence of evolutionary algorithms, in this paper, GA and PSO are strong combined for solving optimal reactive power problem. Firstly, some improved mechanisms such as non-linear ranking selection, competition and selection among several crossover offspring and adaptive change of mutation scaling are adopted in the genetic algorithm. Then, the genetic algorithm is combined with PSO that is improved by dynamical parameters. Proposed algorithm has been tested in standard IEEE 57 bus test system and simulation results reveal the better performance of the proposed algorithm in reducing the real power loss.

\section{References}


[1] O.Alsac, and B. Scott, “Optimal load flow with steady state security”,IEEE Transaction. PAS -1973, pp. 745-751.

[2] Lee K Y ,Park Y M , Oritz J L -A united approach to optimal real and reactive power dispatch , IEEE Transactions on power Apparatus and systems 1985: PAS-104 : 1147-1153

[3] A.Monticelli , M .V.F Pereira ,and S. Granville , "Security constrained optimal power flow with post contingency corrective rescheduling", IEEE Transactions on Power Systems :PWRS-2, No. 1, pp.175-182.,1987.

[4] DeebN ,Shahidehpur S.M ,Linear reactive power optimization in a large power network using the decomposition approach. IEEE Transactions on power system 1990: 5(2) : 428-435

[5] E. Hobson ,'Network consrained reactive power control using linear programming, ' IEEE Transactions on power systems PAS -99 (4) ,pp 868=877, 1980

[6] K.Y Lee ,Y.M Park , and J.L Oritz, "Fuel -cost optimization for both real and reactive power dispatches", IEE Proc; 131C,(3), pp.85-93.

[7] M.K. Mangoli, and K.Y. Lee, "Optimal real and reactive power control using linear programming" , Electr.PowerSyst.Res, Vol.26, pp.1-10,1993.

[8] C.A. Canizares , A.C.Z.de Souza and V.H. Quintana , "Comparison of performance indices for detection of proximity to voltage collapse ,' vol. 11. no.3 , pp.1441-1450, Aug 1996 .

[9] S.R.Paranjothi , andK.Anburaja, "Optimal power flow using refined genetic algorithm", Electr.PowerCompon.Syst, Vol. 30, 1055-1063,2002.

[10] D. Devaraj, and B. Yeganarayana, "Genetic algorithm based optimal power flow for security enhancement", IEE proc-Generation.Transmission and. Distribution; 152, 6 November 2005.

[11] A.Berizzi, C. Bovo, M. Merlo, and M. Delfanti, "A ga approach to compare orpf objective functions including secondary voltage regulation," Electric Power Systems Research, vol. 84, no. 1, pp. 187 $-194,2012$.

[12] C.-F. Yang, G. G. Lai, C.-H. Lee, C.-T. Su, and G. W. Chang, "Optimal setting of reactive compensation devices with an improved voltage stability index for voltage stability enhancement," International Journal of Electrical Power and Energy Systems, vol. 37, no. 1, pp. 50 - 57, 2012.

[13] P. Roy, S. Ghoshal, and S. Thakur, "Optimal var control for improvements in voltage profiles and for real power loss minimization using biogeography based optimization," International Journal of Electrical Power and Energy Systems, vol. 43, no. 1, pp. 830 - 838, 2012.

[14] B. Venkatesh, G. Sadasivam, and M. Khan, "A new optimal reactive power scheduling method for loss minimization and voltage stability margin maximization using successive multi-objective fuzzy lp technique," IEEE Transactions on Power Systems, vol. 15, no. 2, pp. 844 - 851, may 2000.

[15] W. Yan, S. Lu, and D. Yu, "A novel optimal reactive power dispatch method based on an improved hybrid evolutionary programming technique," IEEE Transactions on Power Systems, vol. 19, no. 2, pp. 913 - 918, may 2004.

[16] W. Yan, F. Liu, C. Chung, and K. Wong, "A hybrid genetic algorithminterior point method for optimal reactive power flow," IEEE Transactions on Power Systems, vol. 21, no. 3, pp. 1163 1169, aug. 2006.

[17] J. Yu, W. Yan, W. Li, C. Chung, and K. Wong, "An unfixed piecewiseoptimal reactive power-flow model and its algorithm for ac-dc systems," IEEE Transactions on Power Systems, vol. 23, no. 1, pp. $170-176$, feb. 2008.

[18] F. Capitanescu, "Assessing reactive power reserves with respect to operating constraints and voltage stability," IEEE Transactions on Power Systems, vol. 26, no. 4, pp. 2224-2234, nov. 2011.

[19] Z. Hu, X. Wang, and G. Taylor, "Stochastic optimal reactive power dispatch: Formulation and solution method," International Journal of Electrical Power and Energy Systems, vol. 32, no. 6, pp. $615-621,2010$.

[20] A.Kargarian, M. Raoofat, and M. Mohammadi, "Probabilistic reactive power procurement in hybrid electricity markets with uncertain loads," Electric Power Systems Research, vol. 82, no. 1, pp. $68-80,2012$. 
[21] Jin, N. and Y. Rahmat-Samii, "Advances in particle swarm optimization for antenna designs: Realnumber, binary, singleobjective and multiobjective implementations," IEEE Trans. Antennas Propagat., Vol. 55, 556-567, 2007.

[22] Lee, K. C. and J. Y. Jhang, "Application of particle swarm algorithm to the optimization of unequally spaced antenna arrays," Journal ofEle ctromagnetic Waves and Applications, Vol. 20, 2001-2012, 2006.

[23] Liu, X. F. Y. B. Chen, Y. C. Jiao, et al., "Modified particle swarm optimization of patch antenna design based on IE3D," Journal of Electromagnetic Waves and Applications, Vol. 21, 1819-1828, 2007.

[24] Robinson, J., S. Sinton, and Y. Rahmat-Samii, "Particle swarm, genetic algorithm, and their hybrids: Optimization of a profiled corrugated horn antenna," IEEE International Symposium on Antennas Propagat., Vol. 1, 314-317, 2002.

[25] Juang, C. F., "A hybrid of genetic algorithm and particle swarm optimization for recurrent netword design,” IEEE Trans. Syst., Man, Cybern. - Part B: Cybern., Vol. 34, 997-1006, 2004.

[26] Chaohua Dai, Weirong Chen, Yunfang Zhu, and Xuexia Zhang, "Seeker optimization algorithm for optimal reactive power dispatch," IEEE Trans. Power Systems, Vol. 24, No. 3, August 2009, pp. 1218-1231.

[27] J. R. Gomes and 0. R. Saavedra, "Optimal reactive power dispatch using evolutionary computation: Extended algorithms,” IEE Proc.-Gener. Transm. Distrib.. Vol. 146, No. 6. Nov. 1999.

*Corresponding author.

E-mail address: gklenin@ gmail.com 